\title{
Conditions for Propagating Synchronous Spiking and Asynchronous Firing Rates in a Cortical Network Model
}

\author{
Arvind Kumar, ${ }^{1}$ Stefan Rotter, ${ }^{2,3}$ and Ad Aertsen ${ }^{1,2}$ \\ ${ }^{1}$ Neurobiology and Biophysics, Institute of Biology III, Albert Ludwigs University, ${ }^{2}$ Bernstein Center for Computational Neuroscience, and ${ }^{3}$ Department of \\ Theory and Data Analysis, Institute for Frontier Areas of Psychology and Mental Health, D-79104 Freiburg, Germany
}

\begin{abstract}
Isolated feedforward networks (FFNs) of spiking neurons have been studied extensively for their ability to propagate transient synchrony and asynchronous firing rates, in the presence of activity independent synaptic background noise (Diesmann et al., 1999; van Rossum et al., 2002). In a biologically realistic scenario, however, the FFN should be embedded in a recurrent network, such that the activity in the FFN and the network activity may dynamically interact. Previously, transient synchrony propagating in an FFN was found to destabilize the dynamics of the embedding network (Mehring et al., 2003). Here, we show that by modeling synapses as conductance transients, rather than current sources, it is possible to embed and propagate transient synchrony in the FFN, without destabilizing the background network dynamics. However, the network activity has a strong impact on the type of activity that can be propagated in the embedded FFN. Global synchrony and high firing rates in the embedding network prohibit the propagation of both, synchronous and asynchronous spiking activity. In contrast, asynchronous low-rate network states support the propagation of both, synchronous spiking and asynchronous, but only low firing rates. In either case, spiking activity tends to synchronize as it propagates, challenging the feasibility to transmit information in asynchronous firing rates. Finally, asynchronous network activity allows to embed more than one FFN, with the amount of cross talk depending on the degree of overlap in the FFNs. This opens the possibility of computational mechanisms using transient synchrony among the activities in multiple FFNs.
\end{abstract}

Key words: recurrent network dynamics; feedforward network; synchrony; synaptic conductance; synfire chain; signal propagation; locally connected random network

\author{
Introduction \\ Sensory information in the brain is processed in multiple stages. \\ In this view of information processing, the information needs to \\ be carried from one cortical area to the next. A simple model for \\ this type of processing is a feedforward network, in which each \\ neuron in a given layer receives multiple synaptic inputs from \\ neurons in the previous layer (Hebb, 1949; Abeles, 1991). The \\ issue of propagation of spiking activity (firing rates or synchrony) \\ in isolated feedforward networks (FFNs) has been addressed in \\ several theoretical studies (Diesmann et al., 1999; Gerstner, 2000; \\ Câteau and Fukai, 2001; Gewaltig et al., 2001; van Rossum et al., \\ 2002; Litvak et al., 2003) and in experiments (Reyes, 2003). Nu- \\ merical simulations of FFNs showed that excitation of the first \\ group of neurons with a volley of near synchronous spikes may \\ result in increased synchrony of the spike volley, which remains

\footnotetext{
Received June 5, 2007; revised March 3, 2008; accepted March 6, 2008.

This work was supported by Deutschen Forschungsgemeinschaft-GraKo, Bundesministerium für Bildung und Forschung Grant 01G00420 to the Bernstein Center for Computational Neuroscience Freiburg and European Union Grant 15879-FACETS. We thank Carsten Mehring, Markus Diesmann, Mark-Oliver Gewaltig, and Clemens Boucsein for helpful discussions. All simulations were performed using the NEST simulation software (www.nest-initiative.org).

This article is freely available online through the J Neurosci Open Choice option.

Correspondence should be addressed to Ad Aertsen at the above address. E-mail: aertsen@biologie.uni-freiburg.de.

A. Kumar's present address: Department of Neuroscience, Brown University, Providence, RI 02912. DOI:10.1523/JNEUROSCI.2542-07.2008

Copyright $\odot 2008$ Society for Neuroscience $\quad 0270-6474 / 08 / 285268-13 \$ 15.00 / 0$
}

stable in subsequent layers (Diesmann et al., 1999). Thus, propagating synchrony in the FFN has been proposed as a model to explain the occurrence of task-related precise spike patterns observed in behaving primates (Abeles et al., 1993; Nicolelis et al., 1995; Riehle et al., 1997; Prut et al., 1998). Asynchronous firing rates can be propagated through the FFN only in a restricted parameter regime (van Rossum et al., 2002), as the significant amount of shared connectivity among the neurons tends to synchronize the activity from one group to the next (Litvak et al., 2003). The transmission of activity in the FFN was found to be strongly influenced by the background noise (Gewaltig et al., 2001; van Rossum et al., 2002). In previous studies, noise was considered to be independent of the activity in the FFN. In a more realistic scenario, in which the FFN is embedded in a cortical network, the activity of the FFN would interact with the background activity in the network. Therefore, it is of key importance to study how activity propagation in the FFN would be affected by states of network activity, and to determine which state of background activity would facilitate spike synchrony and/or rate based coding in the FFN. The importance of these issues was emphasized in two previous review papers on the subject (Vogels et al., 2005; Destexhe and Contreras, 2006). In addition, it is necessary to understand how the nonrandom nature of the FFN connectivity would influence the statistics and stability of the ongoing network activity. Previously, it was shown that embedding structures with a high degree of shared connectivity, like an 
FFN, may introduce instability in the network (Mehring et al., 2003).

Here, we study the dynamics of activity propagation in an FFN embedded in a locally connected random network of spiking neurons with conductance-based synapses. We show that synchronous activity traveling in an embedded FFN does not destabilize the dynamics of the network, in contrast to what happens in networks with current-based synapses. For specific states of the background activity, both asynchronous firing rates and transient synchrony can be propagated. However, for most other network activity states, the background activity facilitates information transmission by transient synchrony only. Development of global synchrony in the network was found to be lethal for either type of coding.

Preliminary results have been presented previously in abstract form (Kumar et al., 2006).

\section{Materials and Methods Models}

\section{Neurons}

The neurons in the network were modeled as leaky integrate-and-fire neurons, with the subthreshold dynamics of the membrane potential $V^{i}(t)$ in neuron $i$ described by the following equation:

$$
C \frac{d}{d t} V^{i}(t)+G_{\text {rest }}\left[V^{i}(t)-V_{\text {rest }}\right]=I_{\text {syn }}^{i}
$$

where $I_{\text {syn }}^{i}$ is the total synaptic input current into neuron $i$, and $C$ and $G_{\text {rest }}$ reflect the passive electrical properties of its membrane at rest $\left(V_{\text {rest }}\right.$ $=-70 \mathrm{mV}$ ). When the membrane potential reached a fixed spike threshold $V_{\text {thresh }}$ above rest, a spike was emitted, the membrane potential was reset to its resting value, and synaptic integration was halted for $2 \mathrm{~ms}$, mimicking the refractory period in real neurons (Tuckwell, 1988).

To introduce heterogeneity into the network, the passive properties $(C$ and $\left.G_{\text {rest }}\right)$ and the spiking threshold $\left(V_{\text {thresh }}\right)$ of the neurons were chosen from normal distributions, with mean values $C=250 \mathrm{pF}, G_{\text {rest }}=16.7 \mathrm{nS}$, and $V_{\text {thresh }}=-55 \mathrm{mV}$, respectively.

\section{Network}

We simulated a network of 50,000 neurons, with 40,000 excitatory and 10,000 inhibitory neurons (see Fig. 1a). The excitatory neurons were arranged on a Cartesian grid of $200 \times 200$, and inhibitory neurons on a grid of $50 \times 50$, with the inhibitory grid inserted into the excitatory grid. Thus, the network model represented a laminar patch of cortical neurons of $0.5 \times 0.5 \mathrm{~mm}$, with a spacing of $2.5 \mu \mathrm{m}$ between adjacent neurons (see Fig. 1). To avoid boundary effects, the network was folded as a torus. Each neuron received on average $K$ inputs from within the network, of which $K_{\text {exc }}=2000 \pm 200$ were randomly chosen from the excitatory and $K_{\text {inh }}=500 \pm 50$ from the inhibitory pool. $K_{\text {exc }}$ and $K_{\text {inh }}$ were drawn independently from a normal distribution. The local connection probability of two pyramidal neurons in rat visual cortex has been described as a function of their cell body distance (Hellwig, 2000) using Gaussian profiles. This kind of distance dependent connectivity description holds within a cortical layer; the connectivity across layers follows more complicated rules and was not incorporated in this study. Thus, the connection probability between any two neurons was set to decrease in a Gaussian manner (see Fig. 1b), such that close neighbors had a higher probability of making a synapse, hence the name locally connected random network (LCRN) (Mehring et al., 2003). The width of the Gaussian describing the connection probability $\left(\sigma_{\text {space }}\right)$ was set to $0.2 \mathrm{~mm}$ for both the excitatory and the inhibitory connections (Marino et al., 2005). In addition to the local connections from within the network, each neuron received $K_{\text {ext }}=2000$ excitatory connections from outside, mimicking both long-range connections within the same cortical area and input from other areas in cortex. The simulations were performed on a parallel computer [IBM (White Plains, NY) Regatta] using a parallelized kernel of NEST (Morrison et al., 2005). The dynamical equations were integrated at a fixed temporal resolution of $0.1 \mathrm{~ms}$.

\section{Synapses}

Synaptic input was modeled by transient conductance changes, using $\alpha$-functions (Jack et al., 1975), described below:

$$
G(t)=\left\{\begin{array}{cc}
t \frac{t}{\tau} e^{-\frac{t}{\tau}} & \text { for } t \geq 0 \\
0 & \text { for } t<0
\end{array} .\right.
$$

Synaptic conductance transients were taken to have a uniform rise time of $\tau=0.33 \mathrm{~ms}$ (Matsumura et al., 1996). In in vitro studies, IPSPs have been reported to be slower than EPSPs (Tarczy-Hornoch et al., 1998; Williams and Stuart, 2003). Nonetheless, we chose to adopt the values for excitatory and inhibitory rise times from the study by Matsumura et al. (1996) because these are the only available data from awake, behaving animals. Moreover, we have shown previously that slower inhibition introduces strong synchrony in the network activity (Kumar et al., 2008), which we tried to avoid here. We refer to the peak amplitude $J$ of the conductance transient at $t=\tau$ as the "strength" of the synapse. Generally, excitatory and inhibitory synapses had different strengths $J_{\text {exc }}$ and $J_{\text {inh }}$ assigned.

Assuming fixed synaptic couplings, the total excitatory conductance $G_{\text {exc }}^{i}(t)$ in neuron $i$ was given by the following:

$$
G_{\text {exc }}^{i}(t)=\sum_{j=1}^{K_{\text {exc }}+K_{\text {ext }}} \sum_{k} g_{\text {exc }}\left(t-t_{k}^{j}-D\right) .
$$

The outer sum runs over all excitatory synapses onto this particular neuron, the inner sum runs over the sequence of spikes arriving at a particular synapse. A uniform transmission delay of $D=2 \mathrm{~ms}$ was imposed for all synapses, in all simulations.

Similarly, the total inhibitory conductance, $G_{\text {inh }}^{i}(t)$, in neuron $i$ was given by the following:

$$
G_{\mathrm{inh}}^{i}(t)=\sum_{j=1}^{K_{\mathrm{inh}}} \sum_{k} g_{\mathrm{inh}}\left(t-t_{k}^{j}-D\right) .
$$

Thus, the total synaptic current into neuron $i$ was as follows:

$$
I_{\text {syn }}^{i}(t)=-G_{\text {exc }}^{i}(t)\left[V^{i}(t)-V_{\text {exc }}\right]-G_{\text {inh }}^{i}(t)\left[V^{i}(t)-V_{\text {inh }}\right],
$$

with $V_{\text {exc }}=0 \mathrm{mV}$ and $V_{\text {inh }}=-80 \mathrm{mV}$ denoting the reversal potentials of the excitatory and the inhibitory synaptic currents, respectively. The ratio

$$
g=\frac{J_{\text {inh }}\left|V_{\text {rest }}-V_{\text {inh }}\right|}{J_{\text {exc }}\left|V_{\text {rest }}-V_{\text {exc }}\right|}=\frac{J_{\text {inh }}}{7 J_{\text {exc }}},
$$

corresponding, approximately, to the ratio of peak amplitudes of IPSPs and EPSPs at rest, was used to parameterize the relative strength of inhibition.

On average, every neuron experienced the same amount of conductance change; however, because the neurons had different passive properties, PSPs were slightly different. The mean unitary EPSP and IPSP amplitude was $0.15 \mathrm{mV}$ at rest, in the absence of synaptic background activity.

\section{Embedding of a feedforward network}

To embed a feedforward network into the LCRN, we randomly chose 10 nonoverlapping groups of excitatory neurons of size $w=300$ each, drawn from Gaussian patches of width $\sigma_{\mathrm{FFN}}=50 \mu \mathrm{m}$. Figure $1 c$ shows the spatial layout of the FFN, with different colors denoting different neuron groups. The 10 groups were fully interconnected in a feedforward manner. We corrected the in-degree of the neurons participating in the FFN, such that on average all neurons had an equal number of connections. Thus, neurons participating in the FFN (from group 2 through 10) each received 300 excitatory connections from the neurons in the preceding group and, on average, 1700 excitatory connections from the LCRN, all connections being equally strong. Thereby, we maintained a balanced connectivity in the network. We also ensured that successive groups were not located further than $\sigma_{\text {space }}$ apart, thereby maintaining 
locality in the network. Yet, the minimum distance between successive groups was $0.1 \mathrm{~mm}$, to avoid overlap between groups. Activity in the FFN was initiated by stimulating all neurons in the first group with a pulse packet (PP), that is, a volley of spikes with a Gaussian shape, characterized by its strength $a$, the number of spikes in the volley, and its temporal dispersion $\sigma$, the SD of the spike timings in the volley (Aertsen et al., 1996; Diesmann et al., 1999).

\section{Data analysis}

Network activity

To characterize the dynamical states of simulated network activity, both at the level of single neurons and populations, we used the following descriptors (Kumar et al., 2008).

Mean firing rate was estimated as the mean spike count per second of the neurons in the network. Because of heterogeneities in the network, some neurons did not contribute any spike to the simulation during the observation interval of $4 \mathrm{~s}$; such nonspiking neurons were excluded when estimating the mean firing rate.

Synchrony in the network was measured by the Fano factor of the population activity: FF[pop] $=\operatorname{Var}[$ pop $] / E[$ pop], where $\operatorname{Var}[$ pop] and $E[$ pop] denote the variance and mean of the firing rates of the neural population. To obtain a good estimate of the population activity, we recorded the spike trains of all neurons in the network and their cumulative activity was binned (bin width, $2 \mathrm{~ms}$ ). A population of independent Poisson processes yields FF[pop] = 1 , any mutual dependence results in an increase of Var[pop] and, hence, of FF[pop].

Irregularity of individual spike trains was measured by the squared coefficient of variation of the corresponding interspike interval (ISI) distribution: $\mathrm{CV}[\mathrm{ISI}]=\mathrm{SD}[\mathrm{ISI}] / \mathrm{E}[\mathrm{ISI}]$, where $\mathrm{SD}[\mathrm{ISI}]$ and $\mathrm{E}[\mathrm{ISI}]$ denote the SD and mean of the ISI distribution, respectively. Low values of CV[ISI] reflect more regular spiking, a clock-like pattern yields $\mathrm{CV}=0$. On the other hand, $\mathrm{CV}=1$ indicates Poisson-type behavior. As a measure for irregularity in the network, we used the average irregularity across all neurons.

\section{Transient synchrony in the FFN}

To characterize the propagation of a PP in an FFN we used the following descriptors.

$a_{\text {last }}$. Transmission of an injected PP in an FFN was called successful if a noticeable trace of the PP was observed in the last group of the FFN. The descriptor $a_{\text {last }}$ measures the number of spikes observed in the last group in a short time window $\left(t_{\mathrm{w}}=10 \mathrm{~ms}\right)$ centered at the expected arrival time of the traveling PP.

$\sigma_{\text {last }} \sigma_{\text {last }}$ describes the temporal spread of the activity $a_{\text {last }}$ in the last group of the FFN.

Survival probability. Propagation of a PP in an FFN is a stochastic process, fluctuations in the background activity may amplify or attenuate the propagation. To estimate the survival probability of a traveling PP we stimulated the first group of the FFN 50 times and measured $a_{\text {last }}$. If $a_{\text {last }}$ crossed a threshold of 100 spikes at low enough temporal dispersion, $\sigma_{\text {last }}$ $\leq 5 \mathrm{~ms}$, we considered it a successful propagation. The ratio of the number of successful propagations and the total number of PP stimulations were used as estimates of the survival probability (Gewaltig et al., 2001).

Signal-to-noise ratio. To be distinguishable by postsynaptic neurons, the amplitude of a traveling PP should be stronger than the background activity (i.e., activity of the neurons not participating in the FFN). In the details, see Materials and Methods.
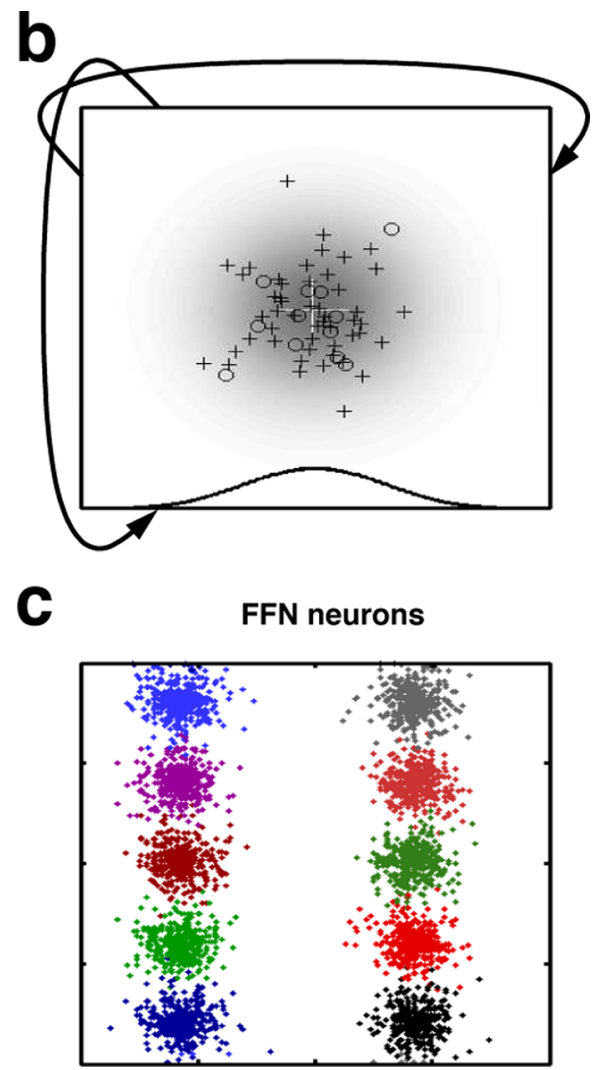

Figure 1. LCRN architecture and embedded FFN. $\boldsymbol{a}$, Network connectivity. Excitatory and inhibitory neurons each receive input of the embedding network that is not explicitly incorporated. The strength of inhibitory synapses $J$. is scaled by a factor $g$ ) and $K_{\text {inh }}$ (inhibitory; marked as $\bigcirc$ ) presynaptic neurons, chosen at random with a probability decreasing with distance (gray shading; solid curve shows cross-section). The boundaries of the network are connected 作), leading to a torus topology. c, In the network, we embedded a fully connected FFN. The panel entitled "FFN neurons" shows the neurons participating in the embedded FFN, with different colors denoting different groups in the FFN. For additional

case of synchronous background activity, it may happen that, although $\mathrm{PP}$ activity reaches the last group, the background is equally strong and synchronized, such that for the postsynaptic neurons the PP and the background activity are indistinguishable. Identifying the background activity with "noise" and the traveling packet with "signal," we defined the signal-to-noise ratio (SNR) as follows:

$$
\mathrm{SNR}=\frac{a_{\mathrm{last}}}{a_{\mathrm{bkg}}}
$$

where $a_{\text {last }}$ is the activity measured in the last group of the surviving PP and $a_{\mathrm{bkg}}$ is the activity in the same time window in another $w=300$ neurons sharing the space with the 10th group, but not participating in the FFN. A high SNR would indicate a stable and discernible propagation of the PP and a low activity background, a low SNR indicates that the PP cannot be distinguished from background, either because the PP was weak or because the background was strong. Next to $a_{\mathrm{bkg}}$, we also estimated $\sigma_{\mathrm{bkg}}$; together, these PP-type parameters were used (compare Fig. 5) to quantify the degree of transient synchrony in the embedding network activity (Aertsen et al., 1996; Diesmann et al., 1999).

\section{Results}

\section{Network dynamics}

A large random network of spiking neurons with current based synapses exhibits distinct activity states, depending on external 

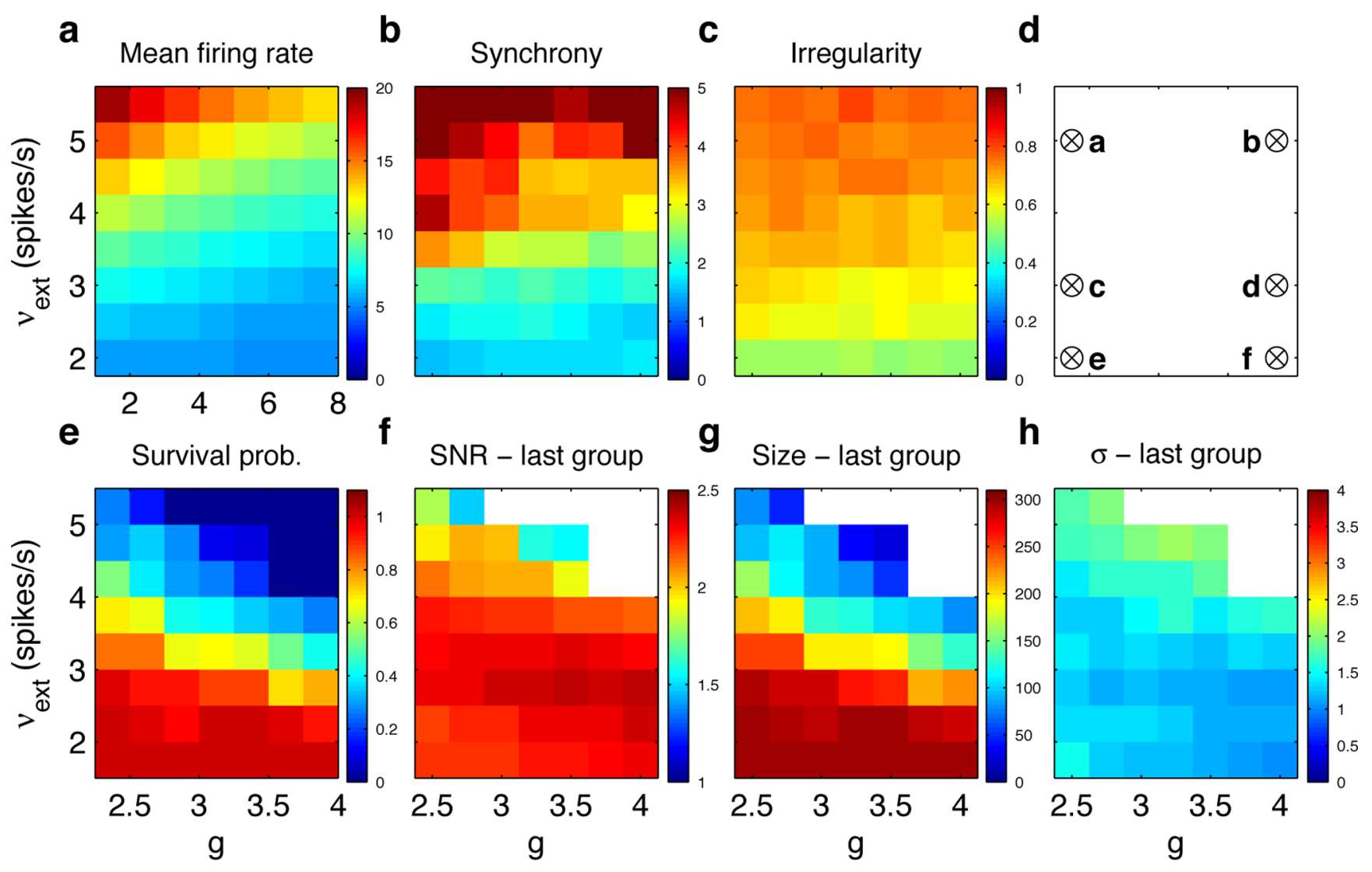

Figure 2. Pulse-packet survival in the embedded FFN as a function of the embedding network activity state. $\boldsymbol{a}$ - $\boldsymbol{c}$ show the mean firing rate, synchrony, and irregularity in the network activity as a function of external input ( $\nu_{\text {ext }}$ ) and inhibition/excitation balance $(g)$. $\boldsymbol{d}$, Six different network activity states, labeled a-f, representative of the spectrum of firing rates, population synchrony, and spiking irregularity exhibited by the network, used as prototype network states in later Figures 5 and $6 . \boldsymbol{e}$, The survival probability strongly depended on the network activity state: higher firing rate and synchrony in the background as well as higher recurrent inhibition destabilized pulse-packet propagation. $f$, When the pulse packet survived, the SNR was always high enough for the pulse packet to be clearly separable from the background activity. $\boldsymbol{g}$ and $\boldsymbol{h}$ show the average state of a surviving pulse-packet ( $a$ and $\sigma$ ) respectively, when it arrived in the last (10th) neuron group. The white space in $\boldsymbol{f}$ - $\boldsymbol{h}$ corresponds to zero survival probability.

excitation $\left(\nu_{\text {ext }}\right)$ and the balance between recurrent inhibition and excitation $g$. The firing pattern of individual neurons can be either regular (R) or irregular (I), population activity can be either synchronous (S) or asynchronous (A). Thus, the network may assume one of four characteristic activity states, viz., AI, SI, AR, or SR (Brunel, 2000; Mehring et al., 2003). Of these, the AI state is considered to best match ongoing cortical network activity in vivo. A random network with conductance based synapses essentially admits the same activity states as observed in networks with current based synapses (Kumar et al., 2008). We have shown previously that in a homogeneous random network, it was not possible to obtain a completely asynchronous population activity, i.e., there are always residual correlations (Kumar et al., 2008). In a homogeneous random network, the residual correlation could be reduced by increasing the size of the network, as a large part of the correlations originates from shared connectivity. In an LCRN, however, increasing the size of the network does not reduce correlations, because the locality of the connectivity rule causes neighboring neurons to share a considerable number of presynaptic inputs. This shared pool of presynaptic neurons is expected to introduce correlations and fast oscillations in the network (Brunel, 2000). These correlations in network activity may be reduced by introducing heterogeneities in the neuronal parameters at various levels (e.g., in neuron properties) (White et al., 1998; Neltener et al., 2000) or in synaptic connections (Denker et al., 2004; Tetzlaff et al., 2005). Here, we introduced heterogeneities in the passive properties and the in-degree of the neu- rons (see Materials and Methods). A distribution of passive properties automatically gives rise to a distribution of the synaptic weights (PSP amplitude) across neurons.

In Figure $2 a-c$ we show the mean firing rate, synchrony and irregularity, respectively, in the network as a function of the external input $\nu_{\text {ext }}$ and the inhibition/excitation balance $g$. Depending on the combination of $\nu_{\text {ext }}$ and $g$, a significant fraction of the neurons remained silent (Kerr et al., 2005), accordingly the network activity descriptors were estimated only for the spiking neurons. We found that with a small degree of heterogeneity it was possible to considerably reduce global synchrony in the network for low $\nu_{\text {ext }}$. Heterogeneities, however, did not eliminate the synchrony in the network activity for stronger external input $\left(\nu_{\text {ext }} \geq\right.$ 4 spikes/s) (Fig. $2 b$ ). Here, the firing rate of the neurons increased, accompanied by an increased synchrony in the population. Further increasing the degree of heterogeneities did not always guarantee more asynchronous activity states. For instance, with a high degree of heterogeneities in the network, part of the neural population could spike at a high rate and in synchrony, whereas the other neurons would be silent (data not shown). The main effect of increased inhibition was to reduce the firing rates (Fig. 2a). Spiking in the network remained largely irregular for all values of $\nu_{\text {ext }}$ and $g$ studied here (Fig. $2 c$ ). Thus, for the parameter range used in the simulations, the network activity belonged to either the SI or AI regime.

Having characterized the dynamics of the spiking activity in the network and its dependence on the prime determinants, the 
external input and the recurrent inhibition/excitation balance, we are now in a position to address the question, how these network dynamics affect the propagation of activity in an FFN embedded in the recurrent network. Based on the results in Figures $2 a-c$, we selected six different network activity states, representative of the spectrum of firing rates, population synchrony and spiking irregularity exhibited by the network activity. These six states are marked as crossed circles, labeled a-f in Figure $2 d$. Here, states a and b represent SI-type activity at high and medium rates, respectively, whereas states c-f represent AI-type activity at medium and low rates, with different degrees of synchrony and irregularity. These six network states will be used in the sequel as representative prototypes to study the impact of network activity on the propagation of activity, both synchronous and asynchronous, in an FFN embedded in the network. For further details on the activity states in conductance based networks and their dependence on external input and recurrent inhibition/excitation balance we refer to our previous work (Kumar et al., 2008).

\section{Propagation of transient synchrony}

LCRNs with current-based synapses have been shown to be very sensitive to transient synchronous input (Mehring et al., 2003). When a group of nearby neurons was forced to spike within a short time window (few milliseconds), the network responded with a "synfire explosion," eventually involving the synchronous spiking of a considerable fraction of the network. This effectively prohibited the stable propagation of synchronized spiking in an embedded FFN. One of the reasons for the synfire explosion to occur is that synapses in that model were implemented as current transients. As a consequence, synchronous PP input evoked a large compound EPSP in the receiving neurons, lasting for $10 \mathrm{~ms}$ or more, governed by the neurons membrane time constant. Because of this large compound EPSP, neurons responded to the PP with more than one spike, effectively amplifying the synchronous input and rapidly giving rise to synchronous spiking in a large fraction of neurons in the network. Such large compound EPSPs, however, are a biologically unrealistic scenario: in real neurons, incoming spikes introduce brief conductance transients. Consequently, a neuron with conductance based synapses would not respond with such large and long-lasting compound PSPs, as the individual PSPs would be attenuated and shortened in the high conductance state (Chance et al., 2002; Kuhn et al., 2004). Based on this observation, it was suggested (Kuhn et al., 2004; Kumar et al., 2008) that conductance based synapses are likely to improve the stability of AI-type network activity state by making it less susceptible to transient input synchrony.

To test this hypothesis, we embedded an FFN with a group size $w=300$ and length 10 into an LCRN with conductance based synapses (Materials and Methods) (Fig. 1c). Transient synchronous input was presented to the network by exciting the first neuron group of the FFN with a pulse packet of strength $a=200$ and width $\sigma=10 \mathrm{~ms}$ (Diesmann et al., 1999). Figure 3 shows the response of the network to such excitation, both in space (Fig. $3 a$ ) and in time (Fig. $3 b$ ). Subsequent panels of Figure $3 a$ show the activity of the neurons at successive intervals of $2 \mathrm{~ms}$. Dots represent spikes from excitatory (blue) and inhibitory (red) neurons at their respective locations within a time interval of $2 \mathrm{~ms}$. Figure $3 b$ shows the propagation of the pulse packet in the time domain for two instances of pulse-packet excitation. Only the neurons participating in the FFN are shown, the color of the dots reflects the group number the corresponding neurons belong to (compare Fig. $1 c$ ). The stimulating PP was centered at $t=215 \mathrm{~ms}$, i.e., after any onset transients of the network dynamics had decayed.
From Figure 3 it is evident that excitation of the embedded FFN had no dramatic effect on the background activity dynamics. In particular, the PP was successfully transmitted to the last group, without recruiting the surrounding neurons into a "synfire explosion."

As can be seen in Figure $3 a$, the traveling PP tends to excite some of its neighboring excitatory and inhibitory neurons after one synaptic delay. We call this after-activity the "halo" of the traveling PP. The size of the halo would depend on the connectivity space constant $\sigma_{\text {space }}$, the group size $w$, and the spatial extent $\sigma_{\mathrm{FFN}}$ of the FFN groups. The interaction of the halo with the background network activity determines the stability and survival probability of PP propagation. A strong halo may interfere or even resonate with the background network dynamics, thereby possibly annihilating or "drowning" the PP activity.

\section{Survival probability of pulse-packet propagation}

In Figure 3, we showed an example of a successful and stable propagation of a PP in an embedded FFN. In that particular case, we tuned the embedding network into an AI state with low firing rate, because it is known from the study of isolated FFNs that the nature of the background activity may strongly influence the survival of the traveling PP (Gewaltig et al., 2001). This problem becomes even more acute when the FFN is truly embedded in a network (as in Fig. 3), because the propagating PP activity itself may affect the background activity, by creating too strong a halo. Thus, depending on the background activity, either the PP may dilute and eventually disappear into the background, or it may grow and/or become very synchronous and, thereby, destabilize the background activity. Between these extremes exists a regime in which the PP propagates in a stable manner, without destabilizing the background. To systematically assess and understand how changes in the network dynamics would affect PP propagation in the FFN, we estimated the survival probability and SNR (see Materials and Methods) of the propagating PP as a function of the embedding network activity state, i.e., as a function of external input $\nu_{\text {ext }}$ and recurrent inhibition/excitation ratio $g$ in the network; the results are shown in Figure 2, $e$ and $f$.

Both the survival probability (Fig. $2 e$ ) and the SNR (Fig. $2 f$ ) of the propagating PP decreased with increasing external input and recurrent inhibition/excitation ratio. The more the network background activity differs from the low-rate AI state, the harder it is for PP activity to survive and be detectable. Yet, the precise dependencies are more complicated, as indicated by the differing shapes of the color gradients in the various panels of Figure 2. Thus, even at a relatively high background firing rate $(\geq 15$ spikes/s), even when synchronous, PP activity in the FFN survived to some extent (Fig. $2 e$, top left corner). The SNR turned out to be a more robust measure than the survival probability: even when the survival probability dropped to values $<0.5$, the PP could still be easily distinguished from background activity (Fig. 2f). Correspondingly, for those PPs that survived, $a_{\text {last }}$ and, especially, $\sigma_{\text {last }}$, remained relatively unaffected by the network activity state (Fig. $2 g, h$, but see Fig. 5). Together, these results indicate that for a wide range of parameters ( $\nu_{\text {ext }}$ and $g$ ), AI-type network activity provides a good substrate for stable PP propagation and, hence, for a scheme of temporal coding relying on it.

\section{Stability of the network activity}

Mehring et al. (2003) showed that LCRNs with current based synapses may become unstable when excited by a transient synchronization. This lead them to conclude that an LCRN with current-based synapses was not a proper substrate for embedding 
a
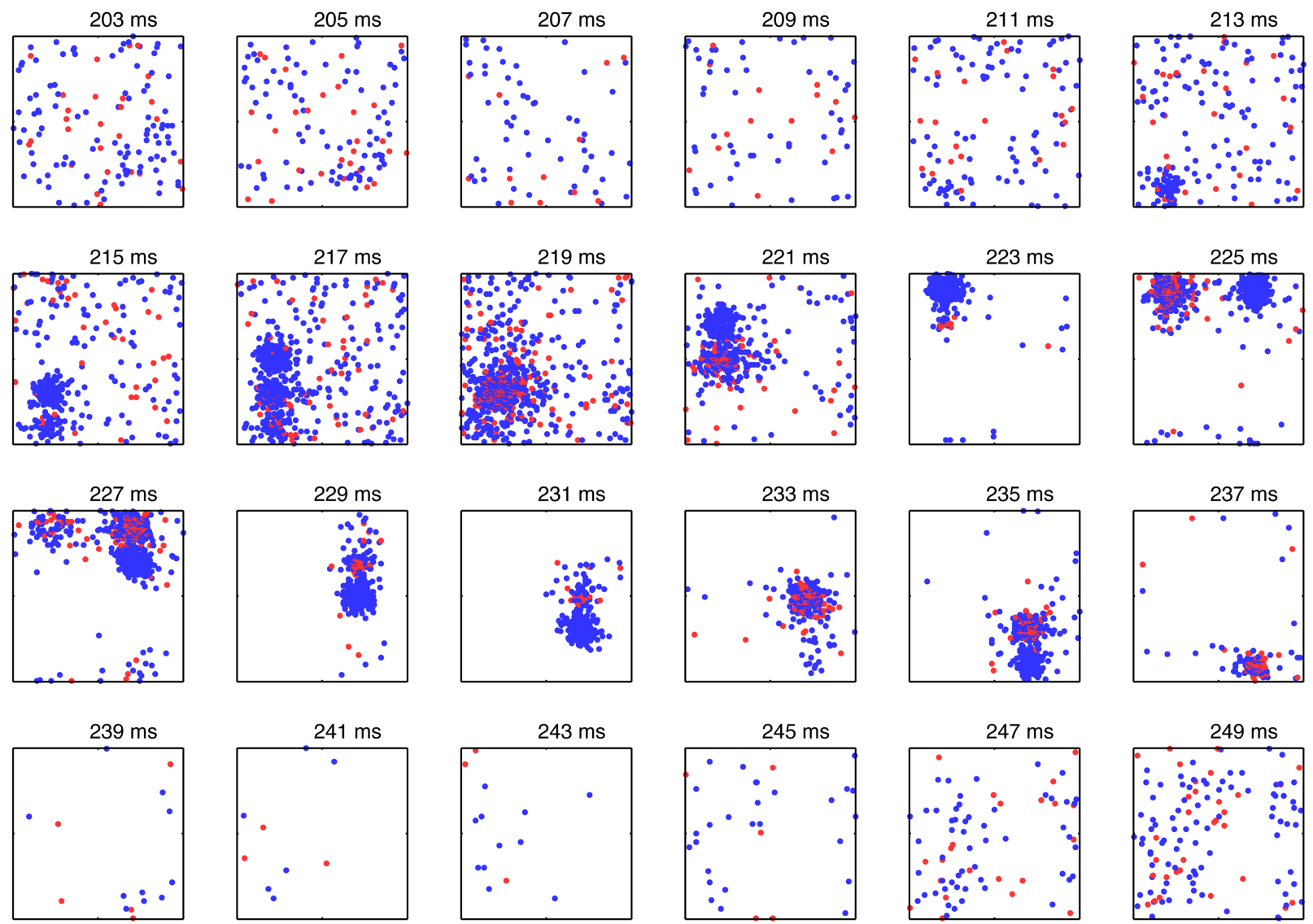

b

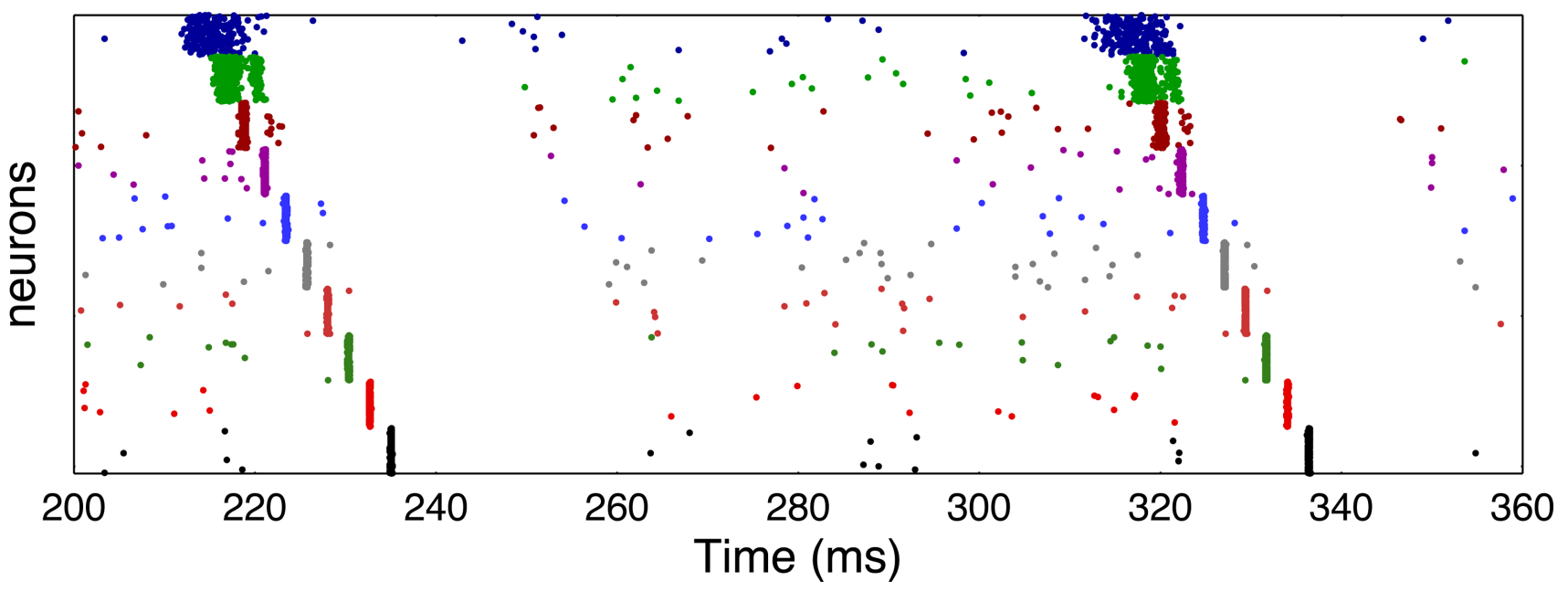

Figure 3. Snap shots of the traveling pulse packet. $\boldsymbol{a}$, Subsequent panels show the spikes of the neurons in successive time frames of $2 \mathrm{~ms}$ duration each. The time of the snapshot is indicated on top of each panel. A dot indicates a spike for a neuron location at that grid-point in the network, with blue referring to excitatory neurons and red to inhibitory ones. A pulse-packet ( $a=200, \sigma=$ $10 \mathrm{~ms}$ ) was injected, with activity peaking at $215 \mathrm{~ms}$. The propagation of the pulse packet is easily seen, the background activity remains stable. The traveling PP tends to excite some of its neighboring excitatory and inhibitory neurons after one synaptic delay: the "halo" of the traveling PP. $\boldsymbol{b}$, Propagation of the pulse packet in the time domain for two instances of pulse-packet transmission. Only spikes of the neurons participating in the FFN are shown; the color of the dots reflects the group number the corresponding neurons belong to (compare Fig. 1c).

FFNs and propagating PPS. Here we showed that when modeling synapses in an LCRN as conductance transients it is indeed possible to reliably propagate pulse packets through the FFN (Fig. 2). We will now consider the differences between these two approaches in more detail.

When synapses are modeled by a current source, PSPs are independent of the synaptic background input impinging on to the neuron. Thus, a neuron with current based synapses in an active network would produce a very large compound EPSP (cEPSP) in response to the incoming PP. The cEPSP would decay with a time constant equal to the membrane time constant (here $20 \mathrm{~ms}$ ). Under the influence of such a strong event, the neuron is likely to spike more than once (refractory period of the neuron is $2 \mathrm{~ms}$ ) and eventually destabilizes the network by recruiting large 
number of neurons to spike in a short time ("synfire explosion") (Mehring et al., 2003).

In contrast, when synapses are modeled as conductance transients, PSPs change with the synaptic background input impinging on the neuron (Chance et al., 2002; Kuhn et al., 2004). Synaptic background activity alters the integrative properties of the neuron by (1) reducing the membrane impedance and, therefore, the effective time constant and (2) by changing the driving force of the synapses. Thus, with increasing synaptic bombardment, these two effects together result in weaker PSPs with a smaller decay time constant (Kuhn et al., 2004). Therefore, a neuron with conductance based synapses, when receiving balanced synaptic activity from the network, would exhibit a smaller and shorter cEPSP when excited with a pulse packet.

To assess the extent of this effect we measured the cEPSP in a neuron under three different conditions (Fig. $4 a-c$ ). First, we recorded the cEPSP in a neuron which participated in the first group of an FFN that was embedded in an LCRN (Fig. $4 a)$. To be able to record a clear cEPSP, we disabled spiking in this particular neuron and stimulated the FFN with a PP ( $a=$ $250, \sigma=10 \mathrm{~ms}$ ). The cEPSP showed a fast overshoot, followed by an undershoot, from the stable state of the membrane potential (at approximately $-58.6 \mathrm{mV}$ ). The rise time and amplitude of the overshoot were governed by the synaptic conductances. The undershoot was because of the activation of inhibitory neurons in the neighborhood (the halo) (Fig. $3 a$ ). Note that the cEPSP reached its peak earlier than the center of the input $\mathrm{PP}(t=0)$ (Fig. $4 a$, black trace).

To demonstrate the effect of synaptic conductances on the cEPSP size and time constant we also performed single-neuron simulations and estimated the effective cEPSP in response to the same PP as before. In addition to the PP, we injected a DC current into the neuron to bring the membrane potential to a stable state of $-58.6 \mathrm{mV}$, i.e., the same value as in Figure $4 a$. Figure $4 b$ (blue trace) shows the average response of a neuron in the absence of background input. The corresponding cEPSP had an amplitude of $\approx 12 \mathrm{mV}$ and a decay time constant of $\approx 15 \mathrm{~ms}$.

In contrast, when the neuron was activated by Poisson-type synaptic background input at 5 spikes/s per synapse, mimicking AI-type activity in the network, the PP stimulation resulted in a cEPSP with a clearly smaller amplitude $(\approx 6 \mathrm{mV})$ and shorter time constant $\approx 5 \mathrm{~ms}$ (Fig. $4 c$, red trace).

The average traces from Figure $4 a-c$ are redrawn in Figure $4 d$ for comparison. Note that the cEPSP in the network (black trace) rises faster than the cEPSPs in the two single neurons (blue and red traces). This faster rise reflects the recurrent excitation from the network impinging on the recorded neuron. The relatively weaker and shorter cEPSP in neurons with conductance-based synapses would be just sufficient to evoke a single spike in the neuron (refractory period, $2 \mathrm{~ms}$ ), thereby excluding the possibil- ity of a phenomenon like the "synfire-explosion" (Mehring et al., 2003) and maintaining stability of the network activity. In contrast, the cEPSP in a neuron with current-based synapses would be similar to the blue trace in Figure $4, b$ and $d$, as in that case the time course of individual PSPs is independent of the synaptic input. Hence, multiple spiking of the neuron in response to PP input is indeed likely, and network instability a predictable result.

\section{The "synfire" attractor}

Studies of the activity dynamics in isolated FFNs with Poissonian background activity showed that stable propagation of a PP primarily depends on the properties of the pulse packet $(a, \sigma)$ and the width $(w)$ of the FFN groups (Diesmann et al., 1999). In particular, it was shown that there exists an attractor, for high $a$ and low $\sigma$, corresponding to stable propagation of PPs. For low $a$ or high $\sigma$, in contrast, the activity diffused and died out after traveling through the first few groups. The location of the separatrix between stable and unstable PP propagation was found to depend on $a, \sigma$, and $w$, and on the background activity (Diesmann et al., 1999; Gewaltig et al., 2001). Note that in these studies, the background activity was assumed to be comprised of independent Poisson processes, quite in contrast to the case of an FFN embedded in an LCRN (Fig. $2 a-c$ ).

Here, we investigated the evolution of the PP in an embedded FFN $(w=300)$ as a function of the PP properties $(a, \sigma)$. We chose six representative network activity states, differing in their firing rates, degree of synchrony and irregularity (Fig. $2 d$ ). For each of 
a

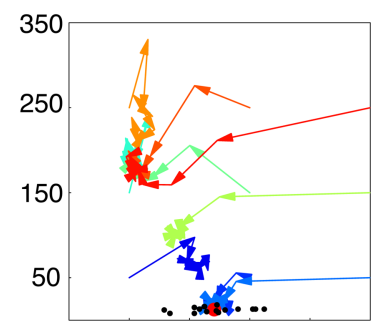

C

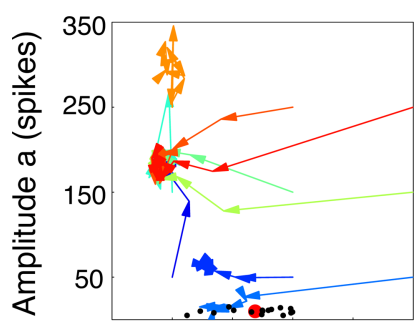

e

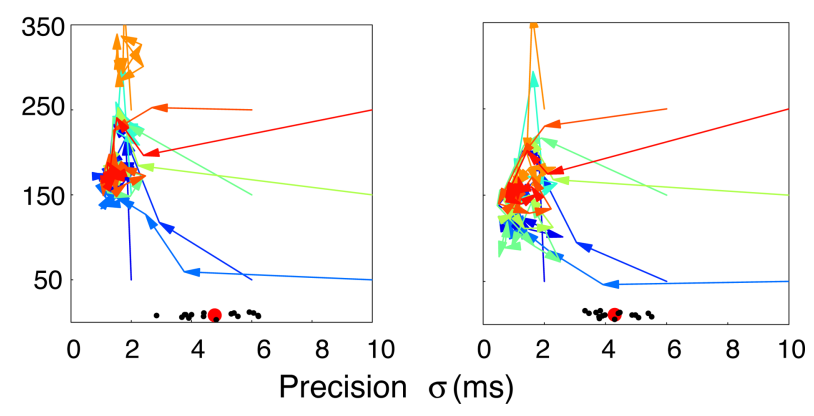

Figure 5. Survival of a PP in the FFN depends on the PP properties. We identified six representative network activity states, marked a-f in Figure $2 d$. $\boldsymbol{a}$, The trajectories show the evolution of the pulse packet as it propagated trough the FFN, embedded in a specific embedding network activity state. $\boldsymbol{a}-\boldsymbol{f}$ correspond to the associated embedding network activity states a-f. Different colors represent pulse packets with different initial conditions. The black dots in each panel show the states of background activity treated as a PP (i.e., $a_{\mathrm{bkg}}$ and $\sigma_{\mathrm{bkg}}$ ) (see Materials and Methods). The big red dot marks the average of the background activity. The PP activity reached its steady state within the first few groups. The PP either converged into a fixed point corresponding to a stable propagation of the PP, or it dissolved in the background activity. Network activity states played an important role in defining the stable fixed point, as is seen by comparing $\boldsymbol{a}-\boldsymbol{f}$. In an Al-type background activity at low firing rates, even very weak PP propagated through the FFN (e). In SI-type background activity, only strong PPs could be propagated $(\boldsymbol{a}, \boldsymbol{b})$.

these six states, we systematically varied $a$ and $\sigma$, and studied the evolution of the PP as it propagated through the FFN. Adopting the approach of Diesmann et al. (1999), the propagation of the PP is displayed as a trajectory in two-dimensional (2D) state space, spanned by $a$ and $\sigma$ (Fig. 5). Each of the six panels, $a-f$, corresponds to the six selected prototypical network activity states (as in Fig. $2 d$ ). The tip of each arrow in a panel marks the state of the $\mathrm{PP}$ in a particular group of the FFN. The background network activity state in the $2 \mathrm{D}$ space is represented by $a_{\mathrm{bkg}}$ and $\sigma_{\mathrm{bkg}}$ (black dots), the big red dots show the average $a_{\mathrm{bkg}}$ and $\sigma_{\mathrm{bkg}}$ (see Materials and Methods).

Observe that for low-firing rate AI network activity, there exists a fixed point in the $(a, \sigma)$-space at $a \approx 150$ and $\sigma \approx 2 \mathrm{~ms}$ (Fig. $5 e, f)$, corresponding to the stable propagation of suitable PPs in an embedded FFN. The basin of attraction (BOA) of the fixed point spans a wide range of $\mathrm{PP}$ parameters. However, as the firing rate and synchrony in the network state increase, the BOA of the fixed point shrinks, and weaker PP start to dissolve in the background (Fig. $5 a-d$ ). Thus, in SI-type background activity and high recurrent inhibition, for all PPs studied, the activity failed to propagate beyond the initial few groups (Figs. $5 b$ ). Also note that as the firing rates increase, the fixed point occurs at higher $a$ (Fig. $5 a, c, e)$. This is a direct consequence of the high conductance state of the network, in which weaker PPs failed to make the postsynaptic neurons spike (Fig. 5, compare $c, e$ ). Finally, we note that in the parameter ranges explored here, we never saw synfire explosions as reported in Mehring et al. (2003).

From Figure 5, we conclude that in synchronous background activity states, transient synchrony may not be propagated. For the network to admit the carrying of temporal information in the form of synchronous spike volleys in embedded FFNs, the background activity must be sufficiently asynchronous and irregular at low enough rates $(\approx 5$ spikes/s). Interestingly, spontaneous activity in the mammalian cortex in vivo is known to be in this regime (Abeles, 1991; Matsumura et al., 1996; Kerr et al., 2005; Waters and Helmchen, 2006).

\section{Propagation of firing rates}

Thus far, we have identified network activity states which support stable PP propagation in an embedded FFN. In this section, we discuss the propagation of firing rates in an FFN embedded in an LCRN as a function of network activity state. Previous work that addressed the propagation of firing rates in isolated FFN (all fully connected, with weak synapses) reported contradictory results (van Rossum et al., 2002; Litvak et al., 2003). Experimental studies have claimed that the development of synchrony would enhance the propagation of firing rates (Reyes, 2003). If that claim holds true, we would expect that in SI states of network dynamics (Fig. 5a,b) firing rates are propagated through the FFN, although a PP may not propagate. Indeed, a previous study showed that firing rates can be propagated in an embedded FFN (Vogels and Abbott, 2005). It should be noted, though, that the latter study considered strongly diluted FFNs, with only $10 \%$ connectivity between successive groups, but with very strong synapses instead. Thus, the typical rate-propagating FFN considered in (Vogels and Abbott, 2005) consisted of a sequence of groups of 33 neurons each, with each neuron in a group receiving inputs from only three neurons in the previous group through enhanced synapses, some 13-fold stronger than non-FFN synapses, each one creating average EPSPs of $8 \mathrm{mV}$ in the active network. In contrast, the typical FFN in our study consisted of groups of 300 neurons, with each neuron in a group receiving inputs from all 300 neurons in the previous group (i.e., a fully connected chain) and all synapses (FFN and non-FFN) being equally weak, with average EPSPs of $0.1 \mathrm{mV}$ in the active network $(0.15 \mathrm{mV}$ at rest) (Kumar et al., 2008). These clear differences in connectivity are expected to have a strong impact on the associated network dynamics. Thus, given the mixed results reported thus far, one cannot easily predict whether firing rates can be faithfully propagated in a fully but weakly connected FFN embedded in a recurrent network. Moreover, understanding the conditions for a possible coexistence of rate and synchrony propagation may help to establish a connection between the two different kinds of neural coding (for review, see Vogels et al., 2005; Destexhe and Contreras, 2006). Therefore, we studied the propagation of asynchronous firing rates in an FFN as a function of the embedding network dynamics in the same systematic manner and covering the identical network activity regimes as we used to characterize the propagation of synchronous spiking (Fig. 5). 
a

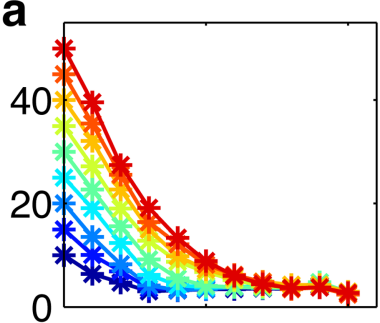

b
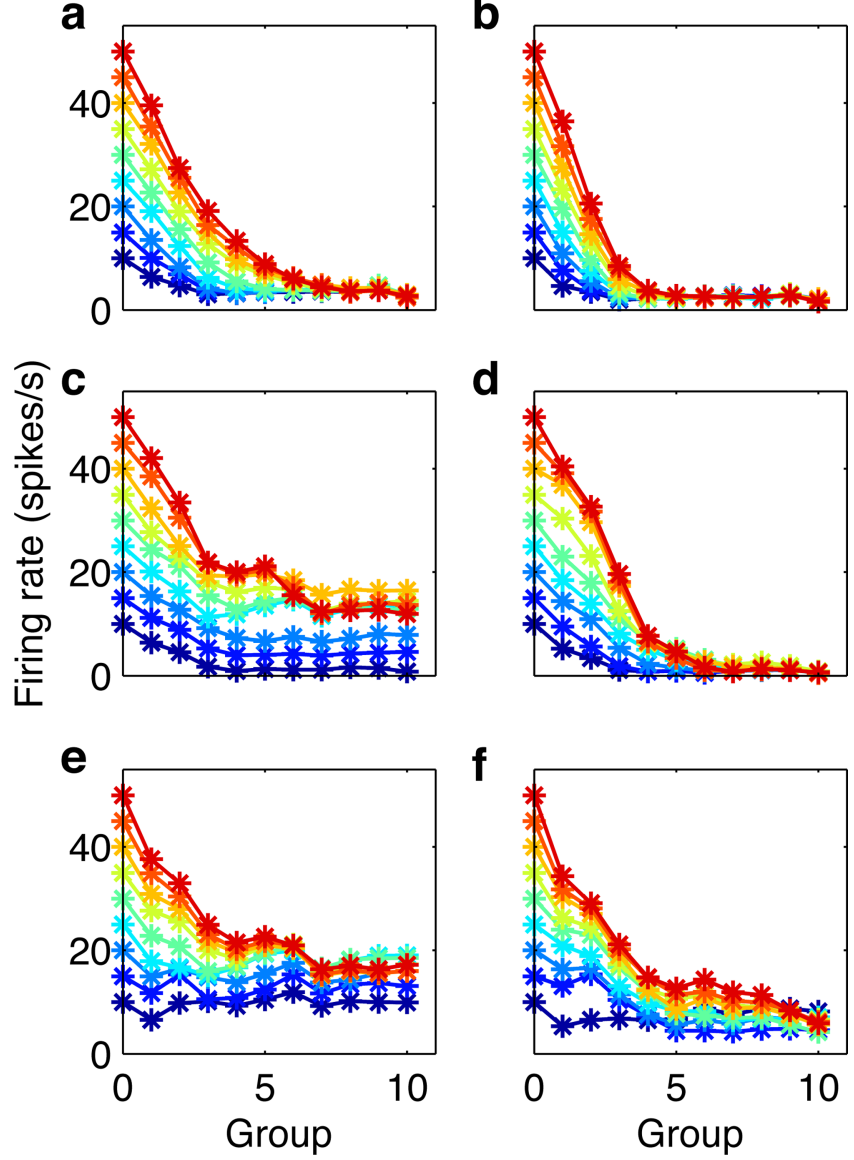

d

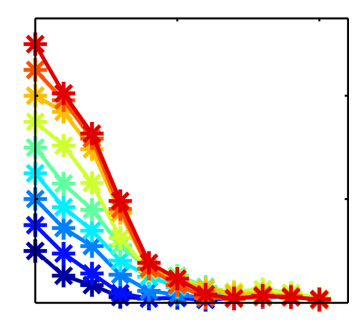

f

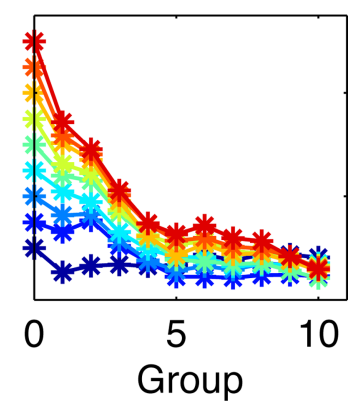

Figure 6. Firing-rate propagation in the FFN as a function of embedding network activity state. The neurons in the first group of the FFN received 200 additional inputs from outside the network for $500 \mathrm{~ms}$, and firing rates were estimated in the subsequent groups. Different color lines indicate a different input rate to the FFN. $\boldsymbol{a}$ - $\boldsymbol{f}$ correspond to the six representative network states marked a-f in Figure $2 d$. The firing rates reached their equilibrium state after traveling through $\sim 5$ groups. Low input rates had a tendency to vanish in the background. Moderately high input rates (15 spikes/s $\leq \mathrm{fr}_{\text {stim }} \leq 35$ spikes $/ \mathrm{s}$ ) were uniquely represented for specific background activity states only $(c, e)$. Input rates above 40 spikes/s converged to low rates, such that a unique representation was not possible. The equilibrium rate depended both on the background firing rate and on the degree of network synchrony. Synchronous background activity states did not support the propagation of input firing rates beyond the initial few groups $(a, d)$.

We stimulated all neurons in the first group of the FFN with asynchronous spiking input $\left(\mathrm{fr}_{\text {stim }}\right)$ over a finite interval. Each neuron received Poisson-type inputs at a given rate (10 spikes/s $\leq$ $\mathrm{fr}_{\text {stim }} \leq 50$ spikes/s) from 200 separate neurons for $500 \mathrm{~ms}$. We estimated the firing rates in the subsequent groups of the FFN as a function of $\mathrm{fr}_{\text {stim }}$ for each of the six representative states of background activity in the network marked a-f in Figure $2 d$. The results are shown in Figure 6. We found that in some cases it was indeed possible to propagate the input firing rates through the FFN up to the last group (Fig. $6 c, e$ ). In general, the firing rate decreased as the activity moved into the later groups. Up to the fourth group, each $\mathrm{fr}_{\text {stim }}$ was uniquely represented in the FFN. However, in the later groups, the response to different input rates for $\mathrm{fr}_{\text {stim }}$ became more and more similar, such that a unique representation of the input was no longer observed. The rate decay was stronger for higher $\mathrm{fr}_{\text {stim. }}$. The embedding network activity played an important role in determining how well firing rates could propagate through the FFN. In low-rate AI states it was possible to propagate input firing rates $\left(10 \mathrm{spikes} / \mathrm{s} \leq \mathrm{fr}_{\text {stim }} \leq\right.$

35 spikes/s) up to the last group (Fig. $6 c, e$ ). In high-rate and synchronous network activity states, it was not possible at all to transmit any input rate beyond the first few groups (Figs. 6a,b). Note that these are precisely the activity states in which the PP also did not survive beyond the initial groups (Fig. $5 a, b$ ). This means that global synchronous states of network dynamics favored neither rate coding nor temporal coding. In contrast, AItype network activity facilitated both rate propagation and PP propagation for low input rates $\mathrm{fr}_{\text {stim }} \leq 30$ spikes/s).

In the scenario where it was possible to transfer firing rates, activity became progressively more synchronous from one group to the next (Fig. 7a). In fact, stimulation of the first group with AI-type activity resulted in SI-type activity in the later groups (Fig. 7b). This emergence of synchrony after stimulation of the first group with Poissonian inputs was caused by the increase in local recurrent input, and it remained local in the neighborhood of the stimulated FFN group. Accidental synchronous events in earlier groups evolved according to the PP attractor dynamics as they propagated to later groups, such that in the 10th group one only observes a sequence of stable pulse packets (Litvak et al., 2003). It is the shared connectivity between successive groups of the FFN that is responsible for the emergence of synchrony in the FFN when stimulated with AI-type input. In fact, only if the degree of shared connectivity from group to group is drastically reduced [e.g., to 10\%, as used by Vogels et al. (2005)], it may be possible to propagate AI-type activity in a limited parameter regime, however, at the expense of having to use unrealistically strong synapses.

\section{Multiple feedforward networks in the LCRN}

Interaction of activity propagating in multiple FFNs embedded in a recurrent network was suggested as a mechanism of feature binding in the cortex (Bienenstock, 1995; Abeles et al., 2004; Hayon et al., 2005). However, for several FFNs to perform feature binding, it is a necessary prerequisite that multiple FFNs may coexist within the same habitat (network area).

To test whether more than one FFN can survive in the networks used in this study, we embedded two FFNs of width $w=$ 300 with five groups each, such that at least one group (here, the third group) of each FFN occupied the same area of the network, and some neurons participated in both FFNs (Fig. 8a,e). We chose the length of the two FFNs to be five, because the fate of a traveling PP was earlier found to be typically determined within the first 3-4 stages (Fig. 5).

In Figure $8, d$ and $h$, we show the coexistence of activity in the FFNs. Both FFNs were simultaneously excited with PPs ( $a=250$, $\sigma=10 \mathrm{~ms}$ ). The spiking activity of the network measured over a time interval of $50 \mathrm{~ms}$ (Fig. $8 d, h$ ) shows that the background remained nearly unchanged while the PPs were propagating. We did not estimate the survival probability of the PP in the two FFNs case, however, we expect it to depend on the background activity in a similar manner as for the single FFN.

\section{Interaction between two FFNs}

In the arrangement shown in Figure $8 a$, the third group of both FFNs occupied a common network area and, in fact, several neurons were shared by both groups. In such an arrangement, excitation of any one of the FFNs resulted in coactivation of the other one, as soon as the propagating PP reached the third group (Fig. $8 b, c)$. In the low-rate AI state, the mean membrane potential of the neurons was $\sim 7 \mathrm{mV}$ away from the spiking threshold. The amplitude of a unitary EPSP in this active state is $\approx 0.1 \mathrm{mV}(\mathrm{Ku}-$ mar et al., 2008). Thus, in this state 70 or more coincident spikes 

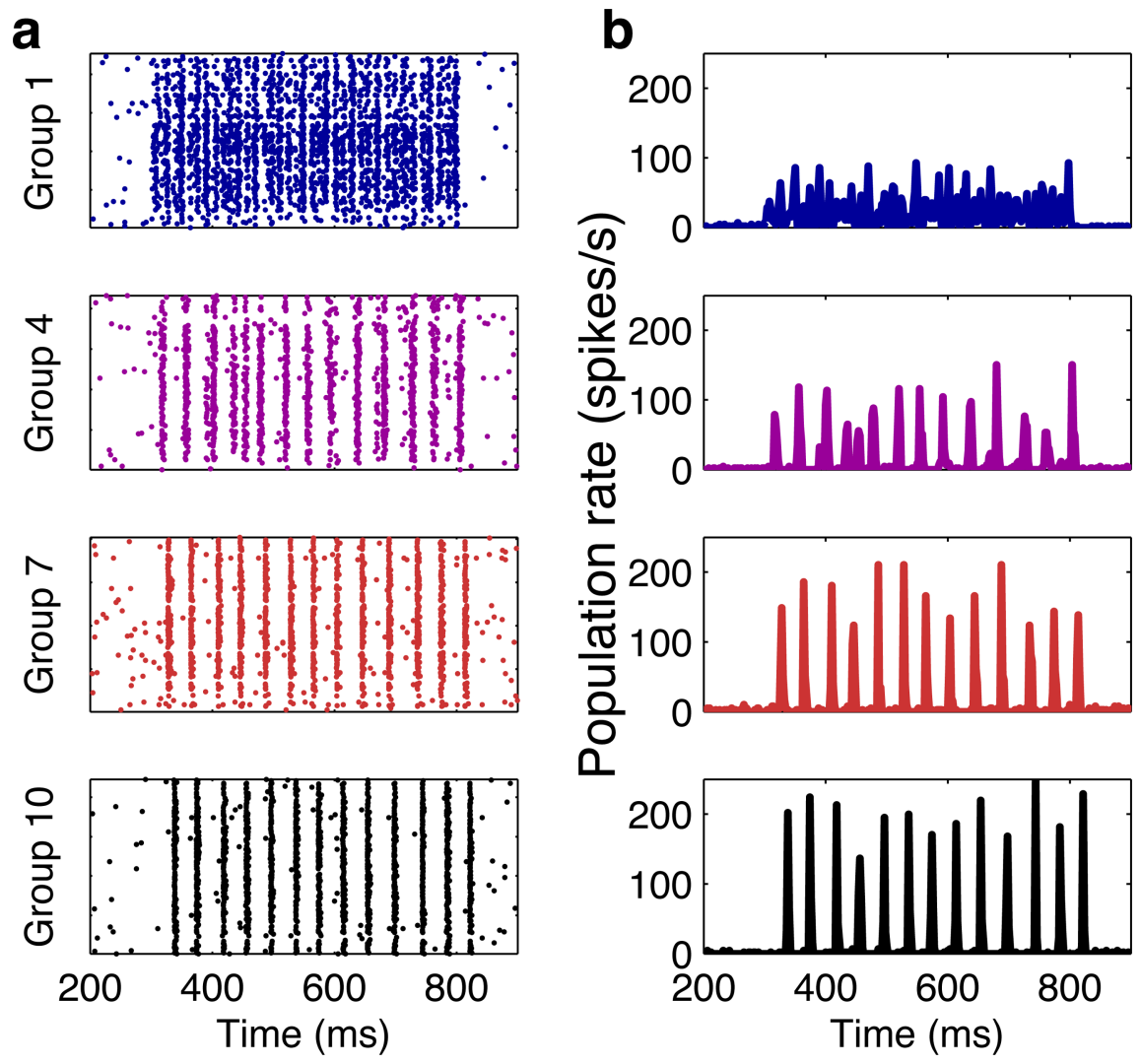

Figure 7. Evolution of synchrony in a rate-stimulated FFN. $\boldsymbol{a}$, The spike trains of the neurons participating in the ratestimulated FFN, embedded in the network activity state labeled c in Figure $2 d$. Neurons are sorted according to their group; only four of the 10 groups are shown, with colors as in Figure 1c. The top-most panel shows the raster of spiking of all neurons belonging to the first group, bottom show the spiking in later groups $(4,7,10)$ of the FFN. All neurons of the first group were activated by Poisson-type spike trains ( 30 spikes/s) for $500 \mathrm{~ms}$, starting at time $300 \mathrm{~ms}$. Only one trial is shown. $\boldsymbol{b}$, Single-trial population rate in high temporal resolution of the spike data in $\boldsymbol{a}$. As the activity propagated through the $\mathrm{FFN}$, it became increasingly synchronous and the population firing rate modulation increasingly transient (almost binary) accordingly.

would be sufficient to induce a spike in the postsynaptic neuron. Therefore, if the overlap between two groups of different chains is $\sim 70$ neurons, the traveling PP in one FFN may ignite the other, partially overlapping FFN. The pool of $\geq 70$ near-coincident spikes may come either from the members of the other FFN or from its halo. This would mean that to avoid cross talk between FFNs, they should (next to sharing only a limited number of neurons) also have nonoverlapping halos. When we separated the third group of the FFNs such that the number of shared neurons was $<70$, we did not observe any cross talk (Fig. $8 f, g$ ). Between the two extremes of full overlap and no overlap, the cross talk between the two FFN would depend on the network activity and parameters of the FFN (data not shown). Given that the simultaneous excitation of two FFNs did not destabilize the background (Fig. 8), nonsimultaneous excitation of the FFNs is even less likely to destabilize the network.

\section{Discussion}

FFNs provide a useful framework to study information processing and propagation of spiking activity in cortical networks (Hebb, 1949; Abeles, 1991; Thorpe and Fabre-Thorpe, 2001). Previous work has shown that under different conditions the FFN can effectively propagate spiking activity, coded either as asynchronous population firing rate (van Rossum et al., 2002; Reyes, 2003) or as transient synchrony (Diesmann et al., 1999; Câteau and Fukai, 2001; Gewaltig et al., 2001). In these studies, back- ground activity was modeled by independent Poisson-type spike trains, assumed to be independent of the activity in the FFN. However, in a realistic scenario the FFN should be embedded in a cortical network, such that the activity in the FFN may affect the background network activity, and vice versa. Also, the background activity in the cortical network can appreciably deviate from Poissonian statistics (Arieli et al., 1996; Brunel, 2000; Mehring et al., 2003; Kumar et al., 2008). Moreover, Mehring et al. (2003) showed that random networks with current-based synapses exhibit an unstable response (synfire explosion) to transient synchronous input. Finally, the highly regular architecture of the FFN would introduce input correlations which could further destabilize the dynamics of the embedding network (Tetzlaff et al., 2002).

Here, we showed, following a suggestion by Kuhn et al. (2004), that modeling synaptic input as conductance transients instead of current injections renders the LCRN stable to transient synchrony. This is not meant to imply that this would be the only way to stabilize the network dynamics. In fact, other proposals made previously have been shown to work as well. Thus, it was suggested to incorporate inhibitory neurons into the FFN (Aviel et al., 2003; Mehring et al., 2003; Hayon et al., 2005), effectively creating a doubly balanced network with a reduced vulnerability to instabilities (Aviel et al., 2003). Such shadow inhibition within the FFN is, in fact, a quite natural extension of the original synfire chain architecture (Abeles, 1991), as it avoids the somewhat artificial need to prohibit inhibitory neurons from appearing in synfire groups. Without such restriction, they would be expected to appear in the same proportion in which they are present in the network at large $(\sim 1-4)$ (Braitenberg and Schüz, 1998). Likewise, it was proposed that the network dynamics can be stabilized by introducing heterogeneities into the network, either in neuron properties (White et al., 1998; Neltener et al., 2000) or in synaptic connections (Denker et al., 2004; Tetzlaff et al., 2005). Adopting such heterogeneities seems, once more, a more biological modeling choice than the artificial restriction of homogeneity in all network parameters. Additional options remain to be explored, including the effect of invoking mixed populations of (distal) current-based and (proximal) conductancebased synapses (Kumar et al., 2004), of having different space constants and/or connectivity profiles of excitatory and inhibitory connections (Holmgren et al., 2003), or of introducing multilayered connectivity schemes (Binzegger et al., 2004; Kremkow et al., 2007), to name just a few. All these options, as the previous ones, present different attempts to move from the approach of studying spiking network dynamics in mathematically convenient random topologies (van Vreeswijk and Sompolinsky, 1996; Brunel, 2000) to biologically more realistic, yet more intricate cortical network architectures. The analysis of the intracellular recordings in Figure 4, however, is indicative of the conditions 

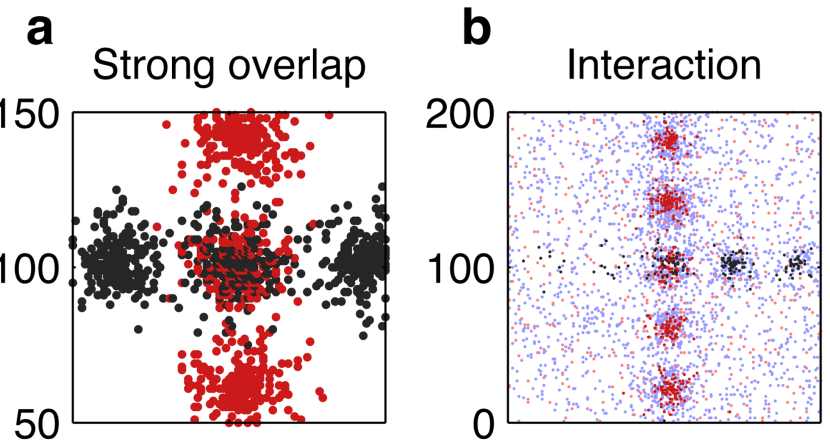

C
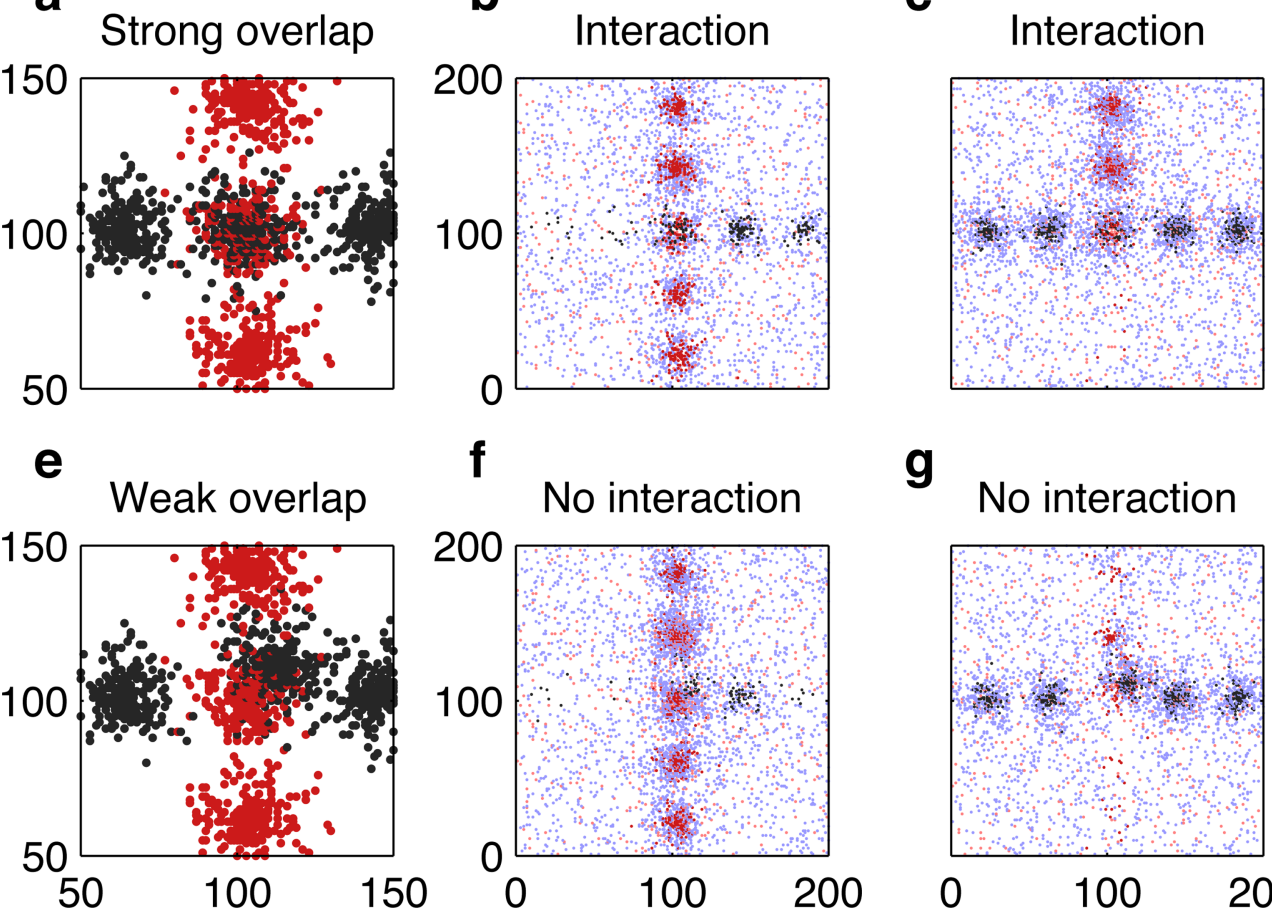

g No interaction

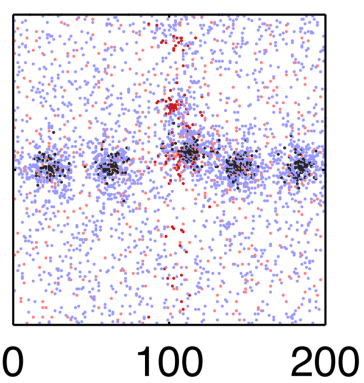

d

\section{Coexistence}

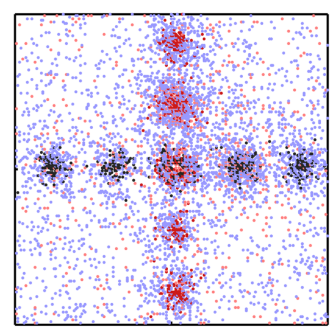

h

\section{Coexistence}

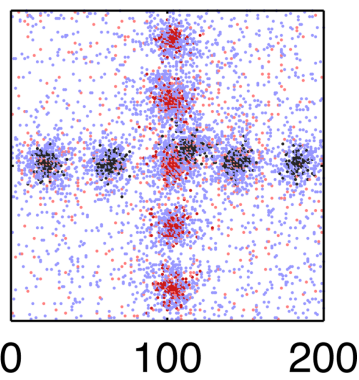

Figure 8. Interaction between multiple FFNs in the LCRN for two different spatial layouts of FFN embedding, differing in the degree of spatial overlap. Both FFNs were composed of five groups each, only the middle three groups (2-4) are shown. $\boldsymbol{a}$ and e show the neurons participating in the two FFNs (brown and black, respectively). $\boldsymbol{b}$ - $\boldsymbol{d}$ and $\boldsymbol{f}$ - $\boldsymbol{h}$ show the spikes of the excitatory neurons over a period of $50 \mathrm{~ms}$ beginning with the initiation of activity in either FFN. Dots indicate spiking of excitatory (blue) and inhibitory (orange) neurons in the embedding network. Spikes elicited by neurons participating in either of the two FFNs are shown in brown or black, respectively. The neurons participating in the FFNs stand out as blobs of activity in these panels. When two embedded FFNs share the same volume at some group, activity of one FFN induces activation of the other one at that group $(\boldsymbol{b}, \boldsymbol{c})$. This cross talk between the two FFNs could be eliminated $(\boldsymbol{f}, \boldsymbol{g})$ by reducing the shared volume of the respective groups $(\boldsymbol{e}) . \boldsymbol{d}, \boldsymbol{h}$, Even when the two FFNs were activated simultaneously, the network remained stable, independent of the degree of overlap between the FFNs.

that need to be met to maintain stability both in the embedded FFN and in the embedding network in response to transient synchronous input. Stable synfire propagation in the embedded FFN will be possible as long as the embedding network admits a lowrate asynchronous irregular activity and the compound EPSPs in the FFN neurons caused by synchronous pulse-packet input do not generate more than one spike per neuron. Network architectures that satisfy these conditions are the prime candidates for stable propagation of synchronous spiking, without the danger of recruiting substantial portions of the embedding network into a synfire explosion.

After characterizing the dependence of the spiking dynamics in the recurrent network on external input and recurrent inhibition/excitation balance (Fig. $2 a-c$ ) and demonstrating the stable embedding of an FFN in an LCRN (Fig. $2 e-h$ ), we went on to address the question of how the recurrent network dynamics affect the propagation of activity, be it synchronous or asynchronous, in an FFN embedded within the network. Based on the results in Figure $2 a-c$, we selected a set of different network activity states, representative of the repertoire of firing rates, population synchrony and spiking irregularity exhibited by the network. This repertoire of network states was then used to study the impact of network activity on the propagation of activity, both synchronous and asynchronous, in the embedded FFN. Results are summarized in Figure 5 for synchronous activity propagation and, likewise, in Figure 6 for asynchronous activity propagation, allowing a direct comparison of the conditions for propagation of both modes of activity for the same repertoire of network activity regimes.

Thus, we studied the propagation of synchronous spiking ac- tivity in the FFN as a function of the ongoing activity in the embedding network. We found that for a large range of parameters it was possible to propagate synchronous PPs without destabilizing the network dynamics (Fig. 5). As in the original work by Diesmann et al. (1999), the state space of PP parameters $(a, \sigma)$ exhibited a stable fixed point at $a=150$ and $\sigma=2 \mathrm{~ms}$, and a failure of stable PP propagation outside the basin of attraction. In low-rate AI states, the basin of attraction of the fixed point was large enough that even weak PPs $(a \approx 100$ and $\sigma \approx 10 \mathrm{~ms}$ ) converged into the fixpoint, resulting in stable propagation through the FFN. As firing rates and synchronization increased in the background network activity, the basin of attraction of the fixed point was reduced. Thus, in network states with strong global synchrony, PP propagation failed.

For the same repertoire of network activity regimes, the propagation of firing rates in the FFN was even more strongly affected by the background network activity (Fig. 6). In fact, only for a small region in the state space of the network, it was possible to propagate firing rates in the FFN for up to 10 groups, albeit only at low input rates $\left(\mathrm{fr}_{\text {stim }} \leq 30 \mathrm{spikes} / \mathrm{s}\right)$; for higher input rates there was no unique representation in the output rate. However, for most activity states it was not possible to propagate the input rate beyond a few initial groups. Moreover, even in the state when elevated firing rates could be detected in the last group, the spiking activity became highly synchronous.

The emergence of synchrony in the rate-stimulated FFN (compare Fig. 7a) did not come as a surprise. It can be attributed to the degree of shared connectivity between successive groups in the FFN. Only if FFNs are sufficiently diluted and intergroup synapses are tuned to be extremely strong, it becomes possible to 
propagate firing rates in an embedded FFN while maintaining asynchrony within groups (Vogels and Abbott, 2005). Given that most synapses in the cortex are quite weak, our observations indicate that in cortical networks firing rate may not transmit information reliably over several processing stages, at least not without at the same time giving rise to strong synchrony. In turn, synchronous input to a single neuron typically induces higher firing rates than asynchronous input (for discussion, see Kuhn et al., 2003). Applying this result to the FFN networks considered in the present study suggests that local firing rates (i.e., within the groups of the FFN) could not increase without causing extra synchrony (as indeed shown in Fig. 7a), and vice versa. It should be stressed that in such a situation it is not sufficient to conceive the firing rate of a single neuron as the low-frequency envelope of its spike density, because that does not adequately represent the coordination of spikes across neurons, crucial for the effects described here. In fact, the high-resolution single-trial population firing rate (Fig. $7 b$ ) is much more informative in this respect, because it captures all spatiotemporal correlations.

Our findings regarding the propagation of firing rates extend the results of an earlier modeling study by Litvak at al. (2003), who showed that if the neuron transfer function is sublinear, it is not possible to transfer firing rates beyond the initial layers. In our model, the neurons had a sublinear and nonmonotonic transfer function (Kuhn et al., 2004). However, our findings contradict previous modeling work (van Rossum et al., 2002; Vogels and Abbott, 2005), for the reasons described above. In fact, our observations showing that in an asynchronously stimulated FFN, the firing rate is propagated only by a few initial few groups before either drowning in the background or developing into strong synchrony are in very good agreement with experimental results (Reyes, 2003). However, note that those experiments were performed on a (virtual) feedforward network in vitro with only few layers. Finally, we point out that the gradual increase of synchronization as firing rates propagate through the FFN may be described by the evolution of rate dynamics and joint ratecorrelation dynamics (Tetzlaff et al., 2004) in FFN architectures.

Global oscillations in the network disturbed or even prevented information transmission based either on asynchronous firing rates or transient synchrony. In contrast, AI-type network activity appears most suited for cortical information transmission in either form. Nevertheless, our results suggest that even in the AI-type background activity state, temporal coding (based on synchronous spiking events) should be a more reliable strategy to propagate information through the network than rate based coding (based on the rate of asynchronous spiking), as in the latter case initial rate differences tend to diminish whereas synchrony tends to increase.

Finally, we showed that in the AI states of background activity it was possible to embed more than one FFN, with the amount of cross talk critically depending on the degree of shared membership in the FFN groups and their halos. This coexistence of multiple FFNs embedded within a single network opens the possibility of a binding mechanism based on transient synchrony among the activities in multiple FFNs.

\section{References}

Abeles, M (1991) Corticonics: Neural circuits of the cerebral cortex, Ed 1. Cambridge, UK: Cambridge UP.

Abeles M, Vaadia E, Bergman H, Prut Y, Haalman I, Slovin H (1993) Dynamics of neuronal interactions in the frontal cortex of behaving monkeys. Concepts Neurosci 4:131-158.

Abeles M, Hayon G, Lehmann D (2004) Modeling compositionality by dynamic binding of synfire chains. J Comput Neurosci 17:179-201.
Aertsen A, Diesmann M, Gewaltig, M.-O (1996) Propagation of synchronous spiking activity in feedforward neural networks. J Physiol (Paris) 90:243-247.

Arieli A, Sterkin A, Grinvald A, Aertsen A (1996) Dynamics of ongoing activity: explanation of the large variability in evoked cortical responses. Science 273:1868-1871.

Aviel Y, Mehring C, Abeles M, Horn D (2003) On embedding synfire chains in a balanced network. Neural Comput 15:1321-1340.

Bienenstock E (1995) A model of neocortex. Netw Comp Neural Syst 6:179-224.

Binzegger T, Douglas RJ, Martin KA (2004) A quantitative map of the circuit of cat primary visual cortex. J Neurosci 39:8441-8453.

Braitenberg V, Schüz A (1998) Cortex: statistics and geometry of neuronal connectivity, Ed 2. Berlin: Springer.

Brunel N (2000) Dynamics of sparsely connected networks of excitatory and inhibitory spiking neurons. J Comput Neurosci 8:183-208.

Câteau H, Fukai T (2001) Fokker-planck approach to the pulse packet propagation in synfire chain. Neural Netw 14:675-685.

Chance FS, Abbott LF, Reyes AD (2002) Gain modulation from background synaptic input. Neuron 35:773-782.

Denker M, Timme M, Diesmann M, Wolf F, Geisel T (2004) Breaking synchrony by heterogeneity in complex networks. Phys Rev Lett 92:074103.

Destexhe A, Contreras D (2006) Neuronal computations with stochastic network states. Science 314:85-90.

Diesmann M, Gewaltig MO, Aertsen A (1999) Stable propagation of synchronous spiking in cortical neural networks. Nature 402:529-533.

Gerstner W (2000) Population dynamics of spiking neurons: fast transients, asynchronous states, and locking. Neural Comput 12:43-89.

Gewaltig MO, Diesmann M, Aertsen A (2001) Propagation of cortical synfire activity: survival probability in single trials and stability in the mean. Neural Netw 14:657-673.

Hayon G, Abeles M, Lehmann D (2005) A model for representing the dynamics of a system of synfire chains. J Comput Neurosci 18:41-53.

Hebb DO (1949) Organization of behavior. A neurophysiological theory. New York: Wiley.

Hellwig B (2000) A quantitative analysis of the local connectivity between pyramidal neurons in layers $2 / 3$ of the rat visual cortex. Biol Cybern $82: 111-121$.

Holmgren C, Harkany T, Svennenfors B, Zilberter Y (2003) Pyramidal cell communication within local networks in layer $2 / 3$ of rat neocortex. J Physiol (Lond) 551:139-153.

Jack JJ, Noble D, Tsien RW (1975) Electric current flow in excitable cells. Oxford: Clarendon.

Kerr J, Greenberg D, Helmchen F (2005) Imaging input and output of neocortical networks in vivo. Proc Natl Acad Sci USA 102:14063-14068.

Kremkow J, Kumar A, Rotter S, Aertsen A (2007) Emergence of population synchrony in a layered network of the cat visual cortex. Neurocomputing 70:2069-2073.

Kuhn A, Aertsen A, Rotter S (2003) Higher-order statistics of input ensembles and the response of simple model neurons. Neural Comput 15:67-101.

Kuhn A, Aertsen A, Rotter S (2004) Neuronal integration of synaptic input in the fluctuation-driven regime. J Neurosci 24:2345-2356.

Kumar A, Kremkow J, Rotter S, Aertsen A (2004) Synaptic integration in a 3 -compartment model of layer 5 pyramidal neurons. FENS Abstr 2:A014.27.

Kumar A, Rotter S, Aertsen A (2006) Stable propagation of synfire activity in locally connected random network. Cosyne Abstr 72.

Kumar A, Schrader S, Aertsen A, Rotter S (2008) High conductance state of cortical networks. Neural Comput 20:1-43.

Litvak V, Sompolinsky H, Segev I, Abeles M (2003) On the transmission of rate code in long feed-forward networks with excitatory-inhibitory balance. J Neurosci 23:3006-3015.

Marino J, Schummers J, Lyon D, Schwabe L, Beck O, Wiesing P, Obermayer $\mathrm{K}$, Sur M (2005) Invariant computations in local cortical networks with balanced excitation and inhibition. Nat Neurosci 8:194-201.

Matsumura M, Chen D, Sawaguchi T, Kubota K, Fetz EE (1996) Synaptic interactions between primate precentral cortex neurons revealed by spike-triggered averaging of intracellular membrane potentials in vivo. J Neurosci 16:7757-7767.

Mehring C, Hehl U, Kubo M, Diesmann M, Aertsen A (2003) Activity dy- 
namics and propagation of synchronous spiking in locally connected random networks. Biol Cybern 88:395-408.

Morrison A, Mehring C, Geisel T, Aertsen A, Diesmann M (2005) Advancing the boundaries of high connectivity network simulation with distributed computing. Neural Comput 17:1776-1801.

Neltener L, Hansel D, Mato G, Meunier C (2000) Synchrony in heterogeneous networks of spiking neurons. Neural Comput 12:1607-1641.

Nicolelis MA, Baccala LA, Lin RC, Chapin JK (1995) Sensorimotor encoding by synchronous neural assembly activity at multiple levels in the somatosensory system. Science 268:1353-1358.

Prut Y, Vaadia E, Bergman H, Haalman I, Hamutal S, Abeles M (1998) Spatiotemporal structure of cortical activity: properties and behavioral relevance. J Neurophysiol 79:2857-2874.

Reyes AD (2003) Synchrony-dependent propagation of firing rate in iteratively constructed networks in vitro. Nat Neurosci 6:593-599.

Riehle A, Grun S, Diesmann M, Aertsen A (1997) Spike synchronization and rate modulation differentially involved in motor cortical function. Science 278:1950-1953.

Tarczy-Hornoch K, Martin KA, Jack JJ, Stratford KJ (1998) Synaptic interactions bewteen smooth and spiny neurones in layer 4 of cat visual cortex in vitro. J Physiol (Lond) 508:351-363.

Tetzlaff T, Geisel T, Diesmann M (2002) The ground state of cortical feedforward networks. Neurocomputing 44-46:673-678.

Tetzlaff T, Morrison A, Geisel T, Diesmann M (2004) Consequences of re- alistic network size on the stability of embedded synfire chains. Neurocomputing 58-60:117-121.

Tetzlaff T, Morrison A, Timme M, Diesmann M (2005) Heterogeneity breaks global synchrony in large networks. In: Proceedings of the 30th Gottingen Neurobiology Conference (Zimmermann H, Krieglstein K, eds), p 206B. Stuttgart, Germany: Thieme.

Thorpe SJ, Fabre-Thorpe M (2001) Neuroscience: seeking categories in the brain. Science 291:312-316.

Tuckwell HC (1988) Introduction to theoretical neurobiology, Vol I. Cambridge, UK: Cambridge UP.

van Rossum MCW, Turrigiano GG, Nelson SB (2002) Fast propagation of firing rates through layered networks of noisy neurons. J Neurosci 2:1956-1966.

van Vreeswijk C, Sompolinsky H (1996) Chaos in neuronal networks with balanced excitatory and inhibitory activity. Science 274:1724-1726.

Vogels TP, Abbott LF (2005) Signal propagation and logic gating in networks of integrate-and-fire neurons. J Neurosci 25:10786-10795.

Vogels TP, Rajan K, Abbott LF (2005) Neural network dynamics. Annu Rev Neurosci 28:357-376.

Waters J, Helmchen F (2006) Background synaptic activity is sparse in neocortex. J Neurosci 26:8267-8277.

White J, Chow C, Ritt J, Soto-Trevino C, Kopell N (1998) Synchronization and oscillatory dynamics in heterogeneous, mutually inhibited neurons. J Comput Neurosci 5:5-16.

Williams SR, Stuart GJ (2003) Voltage- and site-dependent control of the somatic impact of dendritic IPSPs. J Neurosci 23:7358-7367. 